\title{
Dalcetrapib raises HDL-cholesterol level, but does not reduce cardiac risk
}

Dalcetrapib, an inhibitor of cholesteryl ester transfer protein (CETP), raised the HDLcholesterol level in patients hospitalized with an acute coronary syndrome, but this change failed to translate into a reduction in cardiovascular events. These data from the dal-OUTCOMES trial were presented at the AHA 2012 Scientific Sessions.

In previous studies, a high level of HDL cholesterol has been associated with a reduced risk of cardiovascular disease. To determine whether raising the HDL-cholesterol level by inhibition of CETP reduces cardiovascular risk, the dal-OUTCOMES researchers randomly allocated 15,871 patients with an acute coronary syndrome to receive either $600 \mathrm{mg}$ of dalcetrapib daily or a placebo, in addition to the best-available care (including statins, other drugs, and revascularization).

At the second prespecified interim analysis, 1,135 primary end-point events (death from coronary heart disease, nonfatal myocardial infarction, ischaemic stroke, unstable angina, or cardiac arrest with resuscitation) had occurred. The HDL-cholesterol level had increased by $4-11 \%$ in the placebo group, and by $31-40 \%$ in patients taking dalcetrapib, from a mean baseline level of $42 \mathrm{mg} / \mathrm{dl}$. Little change occurred in the LDL-cholesterol level. No difference was observed in the rate of the primary end point $(8.0 \%$ vs $8.3 \%$, respectively; HR 1.04, 95\% CI 0.93-1.16, $P=0.52$ ) and, thus, the trial was terminated early (median follow-up 31 months).

Other CETP inhibitors-evacetrapib and anacetrapib - are undergoing phase III testing. These drugs might raise HDLcholesterol and lower LDL-cholesterol levels more than dalcetrapib, and have a beneficial impact on clinical outcomes.

Gregory B. Lim

Original article Schwartz, G. G. et al. Effects of dalcetrapib in patients with a recent acute coronary syndrome. N. Engl. J. Med. doi:10.1056/NEJMoa1206797 